\title{
A CMOS digital step X-type attenuator with low process variations
}

\author{
Jiayue Wan, Zhiming Chen, and Xinghua Wanga) \\ Beijing Institute of Technology, Haidian distric, Beijing 100081, China \\ a)89811@bit.edu.cn
}

\begin{abstract}
In this paper, a digital step X-type attenuator with low process variations is demonstrated using $90 \mathrm{~nm}$ CMOS technology. The X-type attenuator uses MOSFETs as voltage controlled resistors, which influenced by process parameters. And a compensation circuit associated with the threshold voltage is employed to mitigate the process influence. The attenuator have a maximum attenuation range of $32 \mathrm{~dB}$ with $0.5 \mathrm{~dB}$ steps. The rms amplitude error and rms phase error are $0.42 \mathrm{~dB}$ and $3.1^{\circ}$ over $23.5-30 \mathrm{GHz}$ respectively, and the insertion losse is $9.77 \mathrm{~dB}$ at $25 \mathrm{GHz}$. The core chip size is $0.48 \mathrm{~mm}^{2}$.
\end{abstract}

Keywords: attenuator, CMOS, low effect of process variation

Classification: Integrated circuits

\section{References}

[1] J. Wagner, et al:: "X-type Attenuator in CMOS with novel control linearization, very low phase variations and automatic matching," EuMIC (2013) 200.

[2] H. Dogan, et al:: "Analysis and design of RF CMOS attenuators," IEEE J. Solid-State Circuits 43 (2008) 2269 (DOI: 10.1109/JSSC.2008.2004325).

[3] J. Bae and C. Nguyen: "A novel concurrent 22-29/57-64-GHz dual-band CMOS step attenuator with low phase variations," IEEE Trans. Microw. Theory Techn. 64 (2016) 1867 (DOI: 10.1109/TMTT.2016.2546256).

[4] B.-H. Ku and S. Hong: "6-Bit CMOS digital attenuators with low phase variations for X-Band phased-array systems," IEEE Trans. Microw. Theory Techn. 58 (2010) 1651 (DOI: 10.1109/TMTT.2010.2049691).

[5] M. Davulcu, et al:: "7-Bit SiGe-BiCMOS step attenuator for X-band phasedarray RADAR applications," IEEE Microw. Wireless Compon. Lett. 26 (2016) 598 (DOI: 10.1109/LMWC.2016.2585565).

[6] K. Gharibdoust, et al.: "A fully integrated 0.18-um CMOS transceiver chip for X-band phased-array systems," IEEE Trans. Microw. Theory Techn. 60 (2012) 2192 (DOI: 10.1109/TMTT.2012.2195020).

[7] S. Sim, et al.: "A compact X-band Bi-directional phased-array T/R chipset in 0.13 um CMOS technology," IEEE Trans. Microw. Theory Techn. 61 (2013) 562 (DOI: 10.1109/TMTT.2012.2227786).

[8] B.-H. Ku, et al.: "CMOS integrated circuits for X-band phased array systems," MWSCAS (2011) 1 (DOI: 10.1109/MWSCAS.2011.6026645).

[9] J. Bae, et al.: "A 10-67 GHz CMOS dual-function switching attenuator with improved flatness and large attenuation range," IEEE Trans. Microw. Theory Techn. 61 (2013) 4118 (DOI: 10.1109/TMTT.2013.2288694). 


\section{Introduction}

The amplitude control circuits which widely used in communication and radar system are often implemented with variable-gain amplifier (VGA) and attenuators. Attenuators due to their zero DC power consumption and superior linearity are preferable to VGAs particularly in wideband applications $[1,2,3,4,5]$. Conventional T-/Pi-type, bridge T-/Pi-type and distributed are the basic topologies for most attenuators and can provide step attenuation by digitally controlling the gate voltages $[4,5,6,7,8,9,10]$. However, those conventional step attenuators have high insertion loss and large circuit size. Therefore, a differential X-type attenuator using MOSFETs as voltage controlled resistors is proposed, which would easily influenced by process parameters [1].

In this paper, a digital step X-type attenuator with low process variations is demonstrated. The compensation circuit can provide a voltage, which is associated with the threshold voltage, to cancel the process influence in the core circuit. This attenuator topology also exhibits low phase variations over attenuation range.

\section{The X-type attenuator with low process variations}

\subsection{Conventional X-type attenuator}

The X-type attenuator consist of four MOSFETs operating in the triode region as voltage controlled resistors, as shown in Fig. 1. Two bias voltages $\mathrm{V}_{\mathrm{GS}}$ and $\mathrm{V}_{\mathrm{GP}}$ are needed to control M1, M2 and M3, M4 respectively. The attenuation is achieved by cancelling the signal with the opposite phase, therefore the minimum attenuation is achieved when $\mathrm{V}_{\mathrm{GS}}$ has peak value and the $\mathrm{V}_{\mathrm{GP}}$ has minimum value at the same time, while the DC potentials of the source and drain are the ground. The inductors parallel connected to the input and output can resonate with the parasitic capacitance to get good return losses in higher frequency.

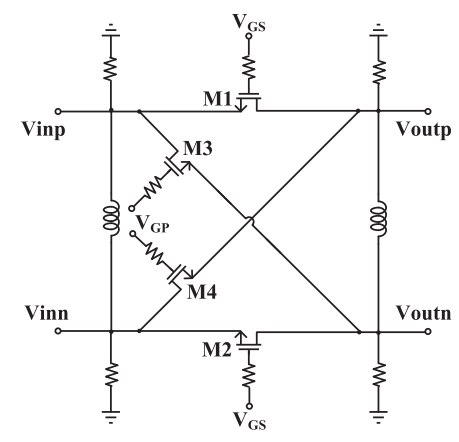

Fig. 1. The schematic of the X-type attenuator

According to the small signal equivalent circuit of the $\mathrm{X}$-type attenuator, the 


$$
S_{21}=\frac{Z_{0}\left(R_{2}-R_{1}\right)}{Z_{0}\left(R_{1}+R_{2}+Z_{0}\right)+R_{1} R_{2}} .
$$

Form the equation above, $S_{21}$ reach the maximum value when $R_{1}$ is low-ohmic and $R_{2}$ is high-ohmic, and $S_{21}$ attain the minimum value when $R_{2}$ equals to $R_{1}$. However, the resistance of an $n$-channel MOSFET transistor operated in the triode region is given by Eq. (2).

$$
R_{o n}=\frac{1}{\mu_{n} C_{o x}\left(\frac{W}{L}\right)_{1}\left(V_{G}-V_{S}-V_{T H 1}\right)} .
$$

The threshold voltage $\mathrm{V}_{\mathrm{TH} 1}$ can be easily influenced by the process parameters.

\subsection{X-type attenuation with compensation circuit to relieve the effect of process variation}

The resistances of MOSFET transistors can be easily influenced by the process variation, which will affact the attenuation value of the X-type attenuator. Therefore, we use a compensation circuit to generate a voltage $\mathrm{V}_{\text {comp }}$, as shown in Fig. 2(a). $V_{\text {comp }}$ is calculated as Eq. (3). Here, I is a constant current.

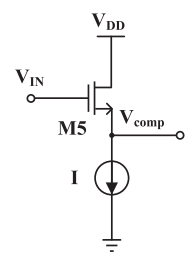

(a)

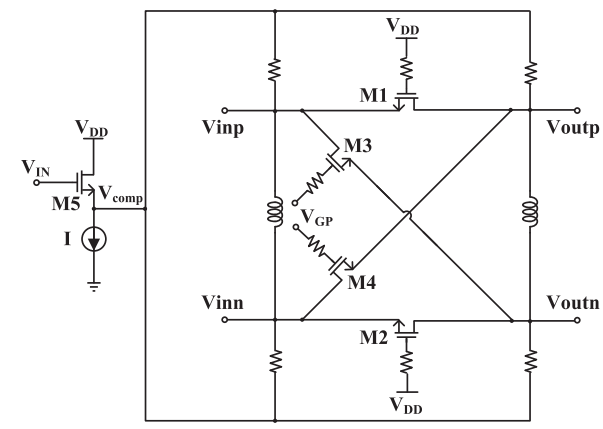

(b)

Fig. 2. The attenuator with compensation circuit.

$$
V_{c o m p}=V_{I N}-V_{T H 2}-\sqrt{\frac{2 I}{\mu_{n} C_{o x}\left(\frac{W}{L}\right)_{2}}} .
$$

When transistors' sizes are the same, $(\mathrm{W} / \mathrm{L})_{1}=(\mathrm{W} / \mathrm{L})_{2}=\mathrm{W} / \mathrm{L}$ and $\mathrm{V}_{\mathrm{TH} 1}=\mathrm{V}_{\mathrm{TH} 2}$. Using $\mathrm{V}_{\text {comp }}$ replace the $\mathrm{V}_{\mathrm{s}}$ in Eq. (2), the influence of the threshold voltage can be cancelled. Therefore, the resistor is now given by Eq. (4).

$$
R_{o n}=\frac{1}{\mu_{n} C_{o x} \frac{W}{L}\left(V_{G}-V_{I N}+\sqrt{\frac{2 I}{\mu_{n} C_{o x} \frac{W}{L}}}\right)} .
$$

Minimum attenuation is achieved when M1 and M2 are lowest-ohmic. Therefore, the gate voltages of M1 and M2 need to be supply voltage while changing the potential of source and drain to control the resistance. The two control voltage are 
$\mathrm{V}_{\mathrm{IN}}$ and $\mathrm{V}_{\mathrm{GP}}$ now, and the $\mathrm{X}$-type attenuation with compensation circuit is shown in Fig. 2(b). The simulation RMS amplitude errors of the conventional X-type attenuator and the proposed one in different process corner is shown in Fig. 3. As we can see, the RMS amplitude errors of both conventional and compensation attenuators are $0.07 \mathrm{~dB}$ in typical process. And the RMS amplitude errors of the convertional attenuator are $1.9 \mathrm{~dB}$ and $2.2 \mathrm{~dB}$ in fast process and slow process at $25 \mathrm{GHz}$, while the bias voltages are the same. And the RMS amplitude errors of the proposed attenuator are $0.3 \mathrm{~dB}$ and $0.36 \mathrm{~dB}$ in fast and slow process at $25 \mathrm{GHz}$ respectively. From the above, the simulation result of the compensation attenuator is far better than the conventional one, so compensation circuit is valid.

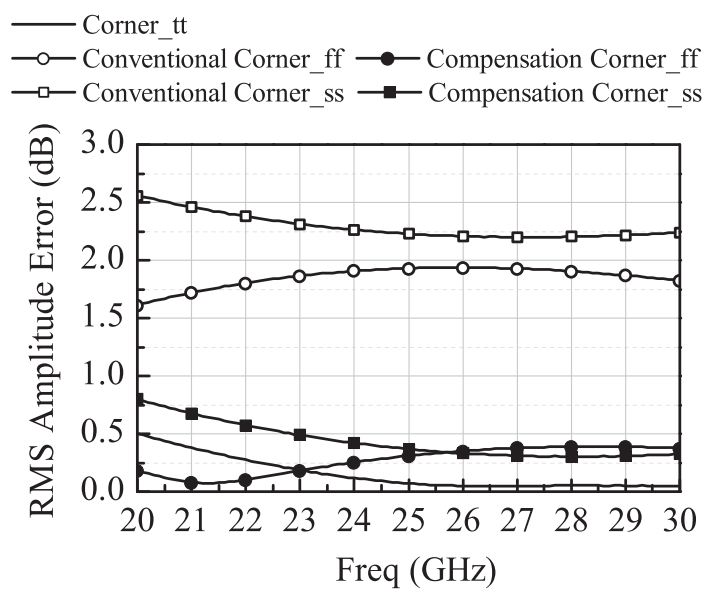

Fig. 3. Simulation RMS amplitude errors of the conventional X-type attenuator and the proposed X-type attenuator in different corner.

\section{Measurement results}

The attenuator was fabricated with an on-chip BGR, DAC, registers and a digital interface for controlling the attenuation. Fig. 4 shows the die photograph of the chip fabricated in a 90-nm CMOS technology.

The main circuit occupies an area of $0.48 \mathrm{~mm}^{2}$, and the total consumption is $10 \mathrm{~mA}$ from a 1.2-V supply. The measurements are performed by on-wafer probing and PCB test board. The measured input and output return losses over the 65 states

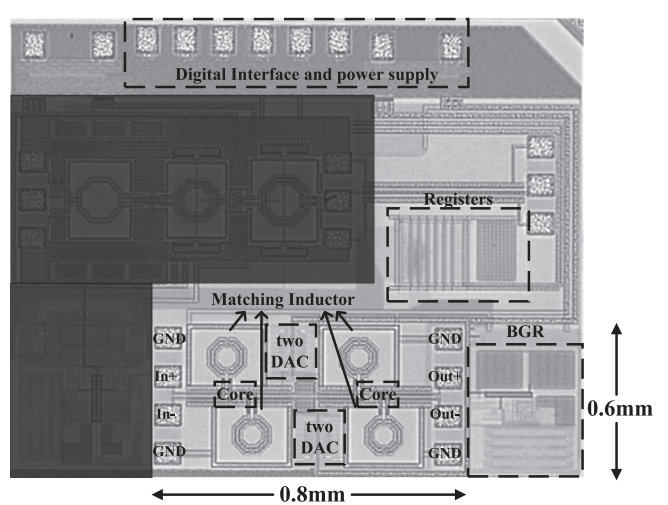

Fig. 4. Die photograph of the proposed attenuator 
are shown in Fig. 5(a) and (b). All the input and output ports are well matched to $100 \Omega$ with measured S11 and S22 better than $10 \mathrm{~dB}$ over $23.5-30 \mathrm{GHz}$.

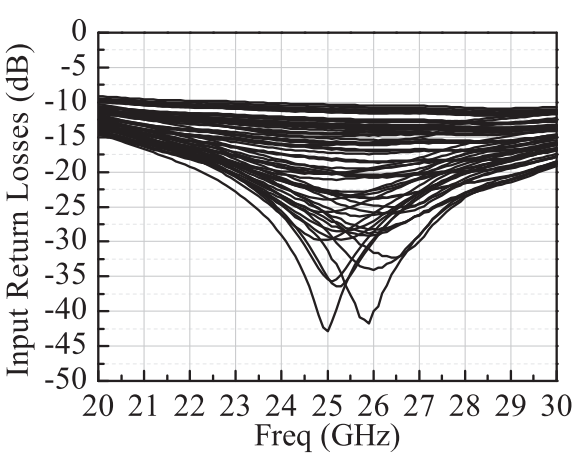

(a)

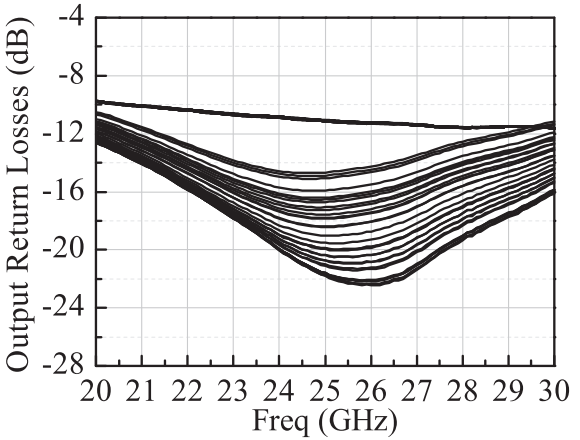

(b)

Fig. 5. Measured performances for all 64 states of the attenuator. (a) input return losses. (b) output return losses.

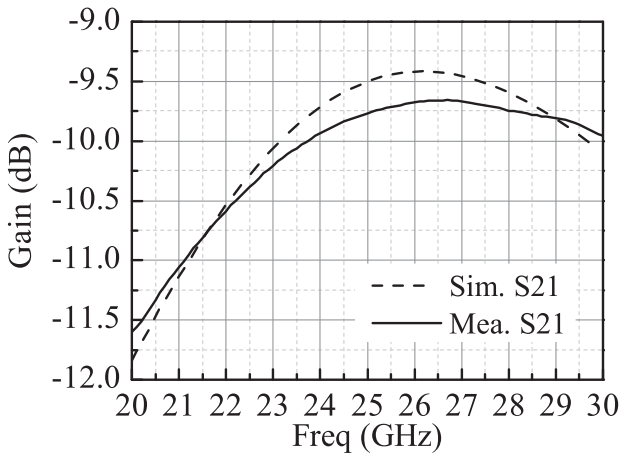

Fig. 6. Measured and simulated insertion losses at the reference state.

Fig. 6 shows the measured insertion losses at the reference state, which is $9.77 \mathrm{~dB}$ at $25 \mathrm{GHz}$ and $9.66-10.1 \mathrm{~dB}$ at $23.5-30 \mathrm{GHz}$.

The measured attenuation relative to the reference state is shown in Fig. 7(a). The maximum attenuation of $32 \mathrm{~dB}$ with LSB of $0.5 \mathrm{~dB}$ is achieved, and the measured attenuation range is $32.15 \mathrm{~dB}$ at $25 \mathrm{GHz}$, and $31.78-32.17 \mathrm{~dB}$ at 23.5-30 GHz. Fig. 7(b) shows the measured insertion phase relative to the reference state. The insertion phase is varying from 4.3 to 13 degree over $23.5-30 \mathrm{GHz}$.

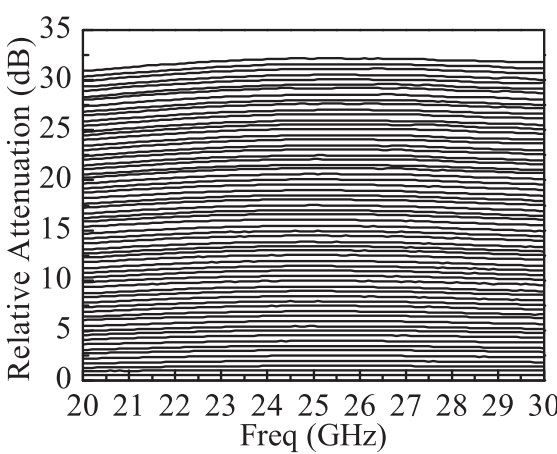

(a)

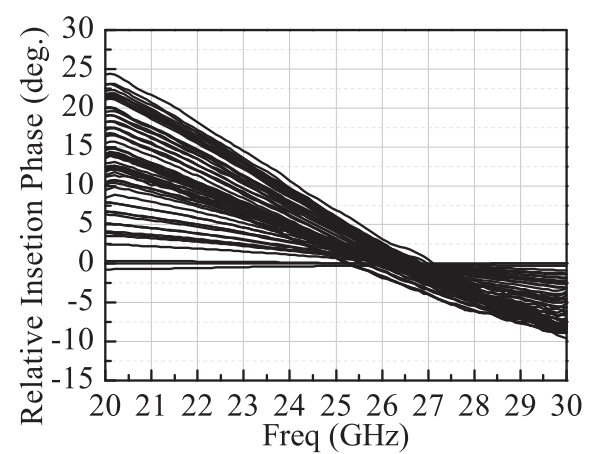

(b) 
Fig. 8 shows the measured rms amplitude and phase errors of the attenuator. The rms amplitude error achieves $0.076 \mathrm{~dB}$ at $25 \mathrm{GHz}$, and it is better than $0.42 \mathrm{~dB}$ over the $23.5-30 \mathrm{GHz}$, which is smaller than half of the LSB steps. The rms phase error is less than $3.1^{\circ}$ at $23.5-30 \mathrm{GHz}$. Table I summarize the performances of the proposed attenuator and compare it with other attenuator designs.

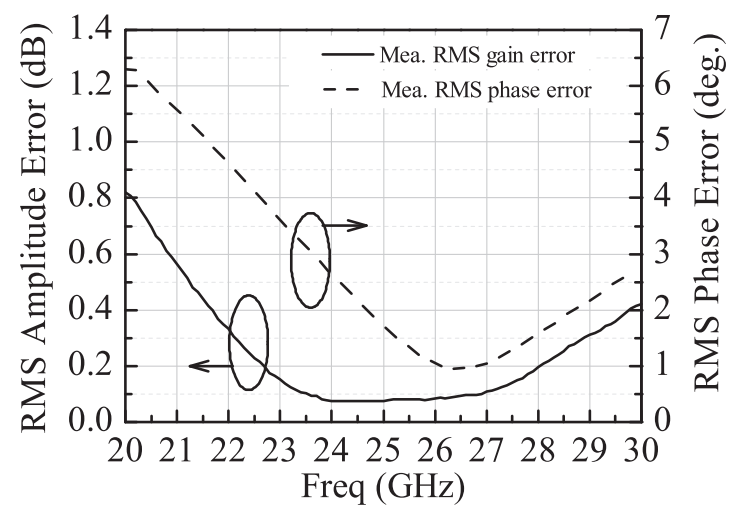

Fig. 8. Measured rms amplitude error and rms phase error.

Table I. Performance comparison with previous works

\begin{tabular}{|c|c|c|c|c|c|}
\hline References & This work & {$[\mathbf{3}]$} & {$[\mathbf{4}]$} & {$[\mathbf{4}]$} & {$[\mathbf{5}]$} \\
\hline Technology & $\begin{array}{c}\mathbf{9 0} \mathbf{~ n m} \\
\text { CMOS }\end{array}$ & $\begin{array}{c}0.18 \mu \mathrm{m} \\
\text { BiCMOS }\end{array}$ & $\begin{array}{c}0.18 \mu \mathrm{m} \\
\text { CMOS }\end{array}$ & $\begin{array}{c}0.18 \mu \mathrm{m} \\
\text { CMOS }\end{array}$ & $\begin{array}{c}0.25 \mu \mathrm{m} \\
\text { BiCMOS }\end{array}$ \\
\hline Frequency $(\mathrm{GHz})$ & $\mathbf{2 3 . 5 - 3 0}$ & $22-29$ & $8-12$ & $8-12$ & $6-12.5$ \\
\hline Att. Range $(\mathrm{dB})$ & $\mathbf{3 2}$ & 16 & 31.5 & 31.5 & 16.51 \\
\hline Insertion Loss $(\mathrm{dB})$ & $\mathbf{9 . 6 6 - 1 0 . 1}$ & $5.4-7.9$ & $8-9.3$ & $9.8-11.3$ & $10.3-12.7$ \\
\hline Rms Amp. error $(\mathrm{dB})$ & $\mathbf{0 . 0 7 - 0 . 4 2}$ & $0.49-0.51$ & $0.2-0.3$ & $0.25-0.4$ & $0.06-0.26$ \\
\hline Rms phase error $(\mathrm{deg})$. & $\mathbf{1 - 3 . 1}$ & $1-4.7$ & $2.6-3.8$ & $2-2.2$ & $2.2-3.5$ \\
\hline Chip Size $\left(\mathrm{mm}^{2}\right)$ & $\mathbf{0 . 4 8}$ & 0.94 & 0.5 & 0.34 & 0.29 \\
\hline
\end{tabular}

\section{Conclusion}

The proposed CMOS digital step X-type attenuator have been designed with a method of alleviating the effect of process variation. The attenuator, fabricated in 90-nm CMOS technology, exhibits less than $0.42 \mathrm{~dB} / 3.1^{\circ} \mathrm{rms}$ amplitude/phase error at $23.5-30 \mathrm{GHz}$. The proposed digital step attenuator is competitive due to its high attenuation range, phase accuracy and wide band of operation.

\section{Acknowledgments}

The author would like to thank Prof. Zhiming Chen in Beijing Institute of technology for the helpful discussion and circuit fabrication. 